\title{
Commentary Prediction of Smoking Cessation with Treatment: The Emerging Contribution of Brain Imaging Research
}

\author{
Arthur L Brody ${ }^{*, 1,2}$ and Francis Joseph McClernon ${ }^{3}$ \\ 'Department of Psychiatry, University of California at Los Angeles, Los Angeles, CA, USA; '2Departments of Psychiatry and Research, \\ VA Greater Los Angeles Healthcare System, Los Angeles, CA, USA; ${ }^{3}$ Department of Psychiatry and Behavioral Sciences, Duke University \\ Medical Center, Durham, NC, USA
}

Neuropsychopharmacology (2015) 40, 1309-1310; doi:10.1038/npp.2015.31

Modern medications for smoking cessation started to become available roughly 35 years ago, with the development and release of nicotine gum. Since that time and with the introduction of other effective smoking cessation medications, approximately 50 studies have been published examining clinical predictors of smoking cessation treatment response. While these clinical predictor studies are helpful in guiding basic aspects of smoking cessation treatment, only recently have studies begun to emerge that demonstrate associations between brain function and smoking cessation treatment response. In this issue of Neuropsychopharmacology, Loughead et al, (2015) report an examination of whether functional magnetic resonance imaging (fMRI) measurements of working memory (WM)-related brain activity could be used to predict smoking relapse above and beyond clinical measures following a brief smoking cessation intervention. Study results link abstinence-induced decreases in left dorsolateral prefrontal cortical (DLPFC) activation and reduced suppression of posterior cingulate cortex (PCC) activity to treatment outcome, thereby implicating the executive control and default mode brain networks, respectively, to the ability to maintain abstinence.

Knowledge of predictors of smoking cessation treatment response is useful in clinical practice. The most commonly replicated clinical predictors of successful smoking cessation include lower levels of nicotine dependence, fewer cigarettes smoked per day, less craving in early abstinence, and high self-efficacy, with other predictors of cessation including high desire to quit, low negative effect, no history of depression, low levels of anger, slow nicotine metabolism, no lapses during early treatment, and lower difficulty reducing smoking over time (for a review, see the introduction to Brody et al, 2014). These predictors are currently most useful for guiding treatment intensity and duration. For example, while a typical smoking cessation treatment course may include serial medication trials and weekly group

\footnotetext{
*Correspondence: Dr Arthur L Brody, Department of Psychiatry, University of California Los Angeles, 300 UCLA Medical Plaza, Suite 2200, Los Angeles, CA 90095, USA, Tel: + I 310478 371 I,

Fax: + I 310206 2802, E-mail: abrody@ucla.edu
}

psychotherapy over a 3 to 4-month course, a smoker with predictors of poor outcome may require combination pharmacotherapy, more intensive and/or frequent psychotherapy, and a longer duration of treatment (eg, 6 months or more). The promise of the study in this month's journal is that an improved understanding of brain function in smokers may in the future be able to influence selection of treatments that affect specific symptoms such as WM deficits or regional brain function such as DLPFC or PCC activation or deactivation.

A clear advance of the Loughead et al study is that it links smoking cessation outcomes with abstinence-induced changes in neural correlates of WM. Extensive prior research demonstrates that smoking abstinence worsens neurocognitive performance on tasks that require the ability to maintain and manipulate stored information over short periods of time (ie, WM). These deficits arise within hours of stopping smoking, may persist for days or weeks, and are resolved upon resumption of smoking (McClernon et al, 2015). Whereas these effects are well documented, how precisely they influence the ability to maintain abstinence remains unclear. For instance, disruption of WM by abstinence may make it more difficult to accomplish everyday tasks, thereby motivating smokers to relapse in order to resolve these effects (ie, negative reinforcement). The ability to maintain abstinence itself requires that smokers continuously monitor their ever-changing environment for potential triggers, keep their motivation to maintain abstinence in mind, and engage in sequences of coping strategies to avoid smoking. All of these processes require WM and as such, the disruption of WM by quitting may make the very act of maintaining abstinence more difficult and less sustainable. In addition to affecting WM itself, smoking abstinence perturbs activation in frontal brain regions during tasks requiring executive function including WM, inhibitory control, and sustained attention. Smoking abstinence also modulates the brain at rest as evidenced by slowing resting electroencephalogram readings and decreasing synchrony between salience, executive control, and default mode networks (Lerman et al, 2014). Thus, while the precise mechanisms by which abstinence- 
induced deficits in WM thwart the smoker's ability to maintain abstinence are unknown, the Loughead et al work strongly suggests that changes in brain activation associated with WM have an important role in smoking cessation.

Despite increased knowledge of the effects of smoking abstinence on brain activity and function, there have been few studies linking neural markers to smoking cessation outcomes, and these studies have generally had small sample sizes. As such, the work in this month's journal, with its relatively large sample size, represents a significant advance in our understanding of what neural outcomes might be prognostic, and in doing so, points to potential therapeutic targets for remediating these deficits. And, while participants in the Loughead et al study received only brief counseling, the study opens the way for investigation of differential prediction of response to other behavioral and pharmacological interventions, which in turn holds promise to inform strategies for personalizing therapy.

The study in this month's journal suggests that further exploration of treatments that affect DLPFC and PCC function is warranted. Most current pharmacological treatments for tobacco dependence affect receptors that are widespread throughout the brain, such as nicotinic acetylcholine receptors or norepinephrine transporters. While these pharmacological treatments improve withdrawal symptoms that could be mediated by DLPFC activity, other more targeted treatments for DLPFC or PCC function could in the future prove useful in treating smokers with poor prognoses. Examples of treatments that could affect specific brain regions include WM training and methods affecting regional neuronal activity, such as repetitive transcranial magnetic stimulation (rTMS) and transcranial direct current stimulation (tDCS), which have been tested for effects on DLPFC function. While results with WM training have been mixed with respect to improving brain function, rTMS to the left DLPFC has shown initial promise in treating tobacco and other drug dependence. In line with the present study, rTMS and tDCS of the DLPFC have been found to significantly improve measures of WM (Brunoni and Vanderhasselt, 2014). Furthermore, a recent study found that rTMS to the DLPFC affected abnormal connectivity within the default mode network, including the PCC (Liston et al, 2014). Thus, the study by Loughead et al indicates that further exploration of treatments that target regional brain activity in smokers may be warranted.

\section{FUNDING AND DISCLOSURE}

Dr Brody is supported by grants from the National Institute on Drug Abuse (R01 DA20872), the Department of Veterans Affairs Office of Research and Development (CSR\&D Merit Review Award I01 CX000412), and the Tobacco-Related Disease Research Program (\#23XT-0002). Dr McClernon is supported by grants from the National Institute on Drug Abuse (P50 DA027840, R01 DA038442, R01 DA025876). The authors declared no conflict of interest.

\section{REFERENCES}

Brody AL, Mukhin AG, Mamoun MS, Luu T, Neary M, Liang L et al (2014). Brain nicotinic acetylcholine receptor availability and response to smoking cessation treatment: a randomized trial. JAMA Psychiatry 71: 797-805.

Brunoni AR, Vanderhasselt MA (2014). Working memory improvement with non-invasive brain stimulation of the dorsolateral prefrontal cortex: a systematic review and meta-analysis. Brain Cogn 86: 1-9.

Lerman C, Gu H, Loughead J, Ruparel K, Yang Y, Stein EA (2014). Large-scale brain network coupling predicts acute nicotine abstinence effects on craving and cognitive function. JAMA Psychiatry 71: 523-530.

Liston C, Chen AC, Zebley BD, Drysdale AT, Gordon R, Leuchter B et al (2014). Default mode network mechanisms of transcranial magnetic stimulation in depression. Biol Psychiatry 76: 517-526.

Loughead J, Wileyto EP, Ruparel K, Falcone M, Hopson R, Gur R et al (2015). Working memory-related neural activity predicts future smoking relapse. Neuropsychopharmacology.

McClernon FJ, Addicott MA, Sweitzer MM (2015). Smoking abstinence and neurocognition: implications for cessation and relapse. In: Munafo MR, Balfour D (eds). The Neurobiology and Genetics of Nicotine and Tobacco. Springer International Publishing: Switzerland. 